\title{
THE GÖEDEL THEOREM AND THE LORENTZ CONTRACTION
}

\author{
Miroslav Pardy \\ Department of Physical Electronics \\ Kotlářská 2, Masaryk University, 61137 Brno, Czech Republic \\ e-mail:pamir@physics.muni.cz
}

August 29, 2019

\begin{abstract}
The power spectral formula of the Cerenkov radiation of the system of two equal charges is derived in the framework of the field theory. The distance between charges is supposed to be relativistically contracted which manifests in the spectral formula. The knowledge of the spectral formula then can be used to verification of the Lorentz contraction of the relativistic length. A feasible experiment for the verification of the Lorentz contraction is suggested.
\end{abstract}

PACS numbers: 03.30.+p, 41.60.Bq

\section{Introduction}

It is well known that the basis of every physical theory is the systems of axioms. From it are drawn the conclusions which are compared with the experiments. Inasmuch in this comparison agreement is found between the messured and the calculated values, the initial system of axioms is considered correct. If no agreement is found, there is good reason for the axioms to be subjected to analysis and corrected.

However, from the theoretical point of view theyselves can be reason for analysis and correction of axioms in themselves. We can ask for their independence, indisputability, for completeness of the syste, or directly for the ideological content of the axioms. From the viewpoint of the ideo-logical content the axiom of the constant light velocity related to the length contraction in the special relativity theory appears to be very important. However such analysis can be incomplete because of the existence of so-called Göedel theorem which is as follows: Göedel theorem: 
In any consistent and rich enough formal structure, based on afinite number of first principles and inference rules, there will always be propositions that may be formulated within this formal system but are undecidable. Such a theory cannot be both consistent and complete. (Ben-Yaacov, 2019). So, let us look on the length contraction and simultaneity from the viewpoint of Euclidean geometry.

So, Let us have two inertial systems $S$ and $S^{\prime}$ of which $S^{\prime}$ moves in the positive direction of axis $x$ so that $x \equiv x^{\prime}$. Let us assume that on each of the $x$-axes of both systems lies a solid rod represented by the abscissa $A B$ in the syste $S$ and $A^{\prime} B^{\prime}$ in $S^{\prime}$ and for their resting length $(A B)_{0},\left(A^{\prime} B^{\prime}\right)_{0}$ holds $(A B)_{0} \equiv\left(A^{\prime} B^{\prime}\right)_{0}$.

Theorem: If $(A B)_{0} \equiv\left(A^{\prime} B^{\prime}\right)_{0}$ in the rest system of $S$ and $v=0$, then it is $(A B)_{0} \equiv$ $\left(A^{\prime} B^{\prime}\right)$, when $v \neq 0$. Proof: The identity of two rods is timeless and non-kinematical notion and it means that the identity of length is timeless and it is only geometrical one. At the same time we can define the simultaneity at points A and B. Namely: if $\left(A \equiv A^{\prime}\right)$, at time $T$ then, $\left(B \equiv B^{\prime}\right)$ at time $T$. This definition of simultaneity differes from the Einstein definition as can be seen in the next text.

A remark: There is a possibility that our statement is undecidable because the Euclidean geometry involves no information on the geometry of moving rod.

\section{The Einstein physical resolution of the Gödel the- orem}

Let us write the text, which is identical with the Einstein text (Einstein, 1916; 1919).

On the Idea of Time in Physics: Lightning has struck the rails on our railway embankment at two places $A$ and $B$ far distant from each other. I make the additional assertion that these two lightning flashes occurred simultaneously. If I ask you whether there is sense in this statement, you will answer my question with a decided "Yes." But if I now approach you with the request to explain to me the sense of the statement more precisely, you find after some consideration that the answer to this question is not so easy as it appears at first sight.

After some time perhaps the following answer would occur to you: "The significance of the statement is clear in itself and needs no further explanation; of course it would require some consideration if I were to be commissioned to determine by observations whether in the actual case the two events took place simultaneously or not." I cannot be satisfied with this answer for the following reason. Supposing that as a result of ingenious considerations an able meteorologist were to discover that the lightning must always strike the places $A$ and $B$ simultaneously, then we should be faced with the task of testing whether or not this theoretical result is in accordance with the reality. We encounter the same difficulty with all physical statements in which the conception " simultaneous " plays a part. The concept does not exist for the physicist until he has the possibility of discovering whether or not it is fulfilled in an actual case. We thus require a definition of simultaneity such that this definition supplies us with the method by means of which, in the present case, he can decide by experiment whether or not both the lightning strokes occurred simultaneously. As long as this requirement is not satisfied, I allow myself to be deceived as a physicist (and of course the same applies if I am not a physicist), when I imagine that I am able to 
attach a meaning to the statement of simultaneity. (I would ask the reader not to proceed farther until he is fully convinced on this point.)

\section{The Čerenkov effect}

The fast moving charged particle in a medium when its speed is faster than the speed of light in this medium produces electromagnetic radiation which is called the Cerenkov radiation.

The prediction of Cerenkov radiation came long ago. Heaviside (1889) investigated the possibility of a charged object moving in a medium faster than electromagnetic waves in the same medium becomes a source of directed electromagnetic radiation. Kelvin (1901) presented an idea that the emission of particles is possible at a speed greater than that of light. Somewhat later, Sommerfeld (1904) proposed the hypothetical radiation with a sharp angular distribution. However, in fact, from experimental point of view, the electromagnetic Cerenkov radiation was first observed in the early 1900's by experiments developed by Marie and Pierre Curie when studying radioactivity emission. In essence they observed the emission of a bluish-white light from transparent substances in the neighborhood of strong radioactive source. But the first attempt to understand the origin of this was made by Mallet (1926; 1929a; 1929b), who observed that the light emitted by a variety of transparent bodies placed close to a radioactive source always had the same bluish-white quality, and that the spectrum was continuous, with no line or band structure characteristic of fluorescence. Unfortunately, these investigations were forgotten for many years. Cherenkov experiments (Čerenkov, 1934) was performed at the suggestion of Vavilov who opened a door to the true physical nature of this effect (Bolotovskii, 2009).

This radiation was first theoretically interpreted by Tamm and Frank (1937) in the framework of the classical electrodynamics. The source theoretical description of this effect was given by Schwinger et al. (1976) at the zero temperature regime and the classical spectral formula was generalized to the finite temperature situation in the framework of the source theory by author (Pardy, 1989). The similar problems was solved and published by author in plenty articles (Pardy, 1983; 2000; 2002; 2004).

The question arises, what is the relation of the Cerenkov radiation to the relativistic length. The relativistic length can be formed by the system of charges of the linear chain, or, only by the two charges of the rest distance $l$. The problem of the radiation of the composed systems of charges is not new and it was defined for the first time in the pioneering work of Ginzburg (1940). Later by Frank $(1942 ; 1946)$, it was given the solution of the problem of the Cerenkov radiation of the electrical and magnetical dipoles oriented parallelly and perpendicularly to the direction of motion. While the parallel orientation gives no surprising result the situation with the perpendicular orientation gives special anomaly which has been frequently discussed in the physical journals. In year 1952 was published the article discussing the Cerenkov radiation of the arbitrary electrical and magnetical multipoles (Frank, 1952). The review of the problems of the Cerenkov radiation of the magnetic and electrical multipoles was given by Frank (1984). The extensive work concerning the radiation by uniformly moving sources is eleborated (Ginzburg, 1986). However, the problem of testing the Lorentz contraction by Cerenkov effect is here considered for the first time (Pardy, 1997; Cavalleri, 2000). 
While the original articles on the Čerenkov radiation involve only determination of the spectral formulae, it is possible interest the question on the relationship between the spectral formula and the Lorentz contraction of the length of some linear object. The specific situation forms the system of two equal or opposite charges of the rest distance $l$. Then, we can expect that the spectral formula of the Cerenkov radiation involves the Lorentz contraction which follows immediately from the Lorentz transformation for coordinates between systems $S^{\prime}$ and $S$ :

$$
x^{\prime}=\gamma(x-v t) ; \quad \gamma=\frac{1}{\sqrt{1-v^{2} / c^{2}}},
$$

where $x$ are coordinates in the system $S$ and $x^{\prime}$ are corresponding coordinates in the system $S^{\prime}$ which is moving with velocity $v$ relative to the system $S$. If the left and right points of the moving rod are $x_{1}, x_{2}$ in the system $S$ and $x_{1}^{\prime}$ and $x_{2}^{\prime}$ in the system $S^{\prime}$, then from equation (1) we have:

$$
x_{2}^{\prime}-x_{1}^{\prime}=\gamma\left(x_{2}-x_{1}\right),
$$

which can be transcribed in the form

$$
a=l \sqrt{1-v^{2} / c^{2}},
$$

where $l$ is the rest length of the rod and $a$ is the length of the moving rod.

The formula (3) is well known and there was general belief since the formulation of the special theory of relativity by Einstein that the so called Lorentz contraction (3) should be visible to the eye. Also Lorentz stated in 1922 that the contraction could be photographed. Similar statements appear in other references concerning the special theory of relativity.

However, the special theory of relativity predicts that the contraction can be observed by a suitable experiment with the nuance that there is distinction between observing and seeing. The situation was analysed for instance by Terrell (1959) and Weisskopf (1960) and others (Dreissler, 2005), who proved that the photograph obtained by an observer depends only on the place and time of taking the picture and is independent of the relative motion of observer and object photographed.

It would be incorrect to state that we see the length contraction, or, that the length "appears" to be contracted by the factor $\sqrt{1-v^{2} / c^{2}}$. As first pointed out by Lampa (1924) and later by Penrose (1959), Terrell (1959) and Weisskopf (1960) what one sees and how an object appears are very different from what is given by the Lorentz contraction. The reason is that various parts of the object are different distances from the observer, and in order for the light rays from the various parts to arrive at the observer at the same time, they must have left the object at different times. It follows from the special theory of relativity that the length contraction is the result of the measurement procedure and the time dilation is also the measurement procedure as was shown by Fok (1961) and author (Pardy, 1969).

In other words, an observation of the shape of a fast moving object involves simultaneous measurement of the position of a number of points on the object. If done by means of light, all the quanta should leave the surface simultaneously, as determined in the observer position at different times. In such observation the data received must be 
corrected for the finite velocity of light, using measured distances to various points of the moving object. In seeing the object, on the other hand, or photographing it, all the light quanta arrive simultaneously at the eye having departed from the object at various earlier times. In such a way this should make a difference between contracted shape which is in principle observable and the actual visual appearance of a fast-moving object. The photograph of a relativistically moving object with a camera using, instead of photons, particles moving much faster than the velocity of light, eliminates the non-desired optical effects and the film would show the object shortened by a factor of $\sqrt{1-v^{2} / c^{2}}$. in the direction of motion. However, such a camera is not physically possible, and we can ask how to correct for the optical effects so that only the relativistic effects will be observed on a photograph taken by an ordinary camera.

In this paper we refer to new approach the measurement of the Lorentz contraction. We use the synchrotron spectrum of the rigid two-body system in such a way, the we read the information on the Lorentz contraction from this spectrum as the proof of the Lorentz contraction.

Obviously, the Čerenkov radiation of the charged two-particle system involves the Lorentz contraction of their rest distance. We will consider the system of two equal charges $e$ which have the mutual rest distance $l$. The Lorentz contraction will be involved in the power spectral formula for this linear system.

In this article we evaluate in source theory the power spectral formula of the Cerenkov radiation of the two-charge system moving with velocity $v$ in the dielectrical medium. Radiative corrections to this two-body Cerenkov radiation are considered too. In conclusion, a feasible experiment is suggested for the verification of the Lorentz contraction.

\section{The field formulation of the problem}

Source theory (Schwinger, et al. 1976; Schwinger, 1970; Dittrich, 1978) is the theoretical construction which uses quantum-mechanical particle language. Initially it was constructed for description of the particle physics situations occurring in the high-energy physics experiments. However, it was found that the original formulation simplifies the calculations in the electrodynamics and gravity where the interactions are mediated by photon or graviton respectively.

The basic formula in the source theory is the vacuum to vacuum amplitude (Schwinger, et al. 1976):

$$
<0_{+} \mid 0_{-}>=e^{\frac{i}{\hbar} W(S)},
$$

where the minus and plus tags on the vacuum symbol are causal labels, referring to any time before and after space-time region where sources are manipulated. The exponential form is introduced with regard to the existence of the physically independent experimental arrangements which has a simple consequence that the associated probability amplitudes multiply and corresponding $W$ expressions add (Schwinger, 1970; Dittrich, 1978).

The electromagnetic field is described by the amplitude (4) with the action

$$
W(J)=\frac{1}{2 c^{2}} \int(d x)\left(d x^{\prime}\right) J^{\mu}(x) D_{+\mu \nu}\left(x-x^{\prime}\right) J^{\nu}\left(x^{\prime}\right),
$$


where the dimensionality of $W(J)$ is the same as the dimensionality of the Planck constant $\hbar$. $J_{\mu}$ is the charge and current densities. The symbol $D_{+\mu \nu}\left(x-x^{\prime}\right)$, is the photon propagator and its explicit form will be determined later.

It may be easy to show that the probability of the persistence of vacuum is given by the following formula (Schwinger, et al. 1976):

$$
\left|<0_{+}\right| 0_{-}>\left.\right|^{2}=\exp \left\{-\frac{2}{\hbar} \operatorname{Im} W\right\} \stackrel{d}{=} \exp \left\{-\int d t d \omega \frac{P(\omega, t)}{\hbar \omega}\right\},
$$

where we have introduced the so called power spectral function (Schwinger, et al. 1976) $P(\omega, t)$. In order to extract this spectral function from $\operatorname{Im} W$, it is necessary to know the explicit form of the photon propagator $D_{+\mu \nu}\left(x-x^{\prime}\right)$.

The electromagnetic field is described by the four-potentials $A^{\mu}(\phi, \mathbf{A})$ and it is generated by the four-current $J^{\mu}(c \varrho, \mathbf{J})$ according to the differential equation (Schwinger, et al. 1976):

$$
\left(\Delta-\frac{\mu \varepsilon}{c^{2}} \frac{\partial^{2}}{\partial t^{2}}\right) A^{\mu}=\frac{\mu}{c}\left(g^{\mu \nu}+\frac{n^{2}-1}{n^{2}} \eta^{\mu} \eta^{\nu}\right) J_{\nu}
$$

with the corresponding Green function $D_{+\mu \nu}$ :

$$
D_{+}^{\mu \nu}=\frac{\mu}{c}\left(g^{\mu \nu}+\frac{n^{2}-1}{n^{2}} \eta^{\mu} \eta^{\nu}\right) D_{+}\left(x-x^{\prime}\right),
$$

where $\eta^{\mu} \equiv(1, \mathbf{0}), \mu$ is the magnetic permeability of the dielectric medium with the dielectric constant $\varepsilon, c$ is the velocity of light in vacuum, $n$ is the index of refraction of this medium, and $D_{+}\left(x-x^{\prime}\right)$ was derived by Schwinger et al. (1976) in the following form:

$$
D_{+}\left(x-x^{\prime}\right)=\frac{i}{4 \pi^{2} c} \int_{0}^{\infty} d \omega \frac{\sin \frac{n \omega}{c}\left|\mathbf{x}-\mathbf{x}^{\prime}\right|}{\left|\mathbf{x}-\mathbf{x}^{\prime}\right|} e^{-i \omega\left|t-t^{\prime}\right|} .
$$

Using formulae (5), (6), (8) and (9), we get for the power spectral formula the following expression (Schwinger et al., 1976):

$$
\begin{aligned}
P(\omega, t)= & -\frac{\omega}{4 \pi^{2}} \frac{\mu}{n^{2}} \int d \mathbf{x} d \mathbf{x}^{\prime} d t^{\prime} \frac{\sin \frac{n \omega}{c}\left|\mathbf{x}-\mathbf{x}^{\prime}\right|}{\left|\mathbf{x}-\mathbf{x}^{\prime}\right|} \cos \left[\omega\left(t-t^{\prime}\right)\right] \times \\
& \times\left\{\varrho(\mathbf{x}, t) \varrho\left(\mathbf{x}^{\prime}, t^{\prime}\right)-\frac{n^{2}}{c^{2}} \mathbf{J}(\mathbf{x}, t) \cdot \mathbf{J}\left(\mathbf{x}^{\prime}, t^{\prime}\right)\right\}
\end{aligned}
$$

Now, we are prepared to apply the last formula to the situations of the two equal charges moving in the dielectric medium.

\section{The Čerenkov radiation of the two-charge system}

It is usually supposed that the Čerenkov radiation in electrodynamics is produced by uniformly moving charge with the constant velocity. Here we consider the system of two equal charges $e$ with the constant mutual distance $a=|\mathbf{a}|$ moving with velocity $\mathbf{v}$ in dielectric medium. We follow the author articles (Pardy, 1997; 2007). 
In this situation the charge and the current densities for this system are given by the by the following equations:

$$
\begin{gathered}
\varrho=e[\delta(\mathbf{x}-\mathbf{v} t)+\delta(\mathbf{x}-\mathbf{a}-\mathbf{v} t)] \\
\mathbf{J}=e \mathbf{v}[\delta(\mathbf{x}-\mathbf{v} t)+\delta(\mathbf{x}-\mathbf{a}-\mathbf{v} t)] .
\end{gathered}
$$

where $\mathbf{a}$ is the vector going from the left charge to right charge with the length of $a=|\mathbf{a}|$ in the system $S$.

Let us suppose that $\mathbf{v}\|\mathbf{a}\| x$. Then, after insertion of eq. (11) and (12) into eq. (10), putting $\tau=t^{\prime}-t$, and $\beta=v / c$, where $v=|\mathbf{v}|$, we get instead of the formula (10) the following relation:

$$
P(\omega, t)=2 P_{1}(\omega, t)+P_{2}(\omega, t)+P_{3}(\omega, t),
$$

where

$$
\begin{gathered}
P_{1}(\omega, t)=\frac{1}{4 \pi^{2}} \frac{e^{2} \mu \omega}{c^{2}} v\left[1-\frac{1}{n^{2} \beta^{2}}\right] \int_{-\infty}^{\infty} d \tau \frac{\sin n \omega \beta \tau}{\tau} \cos \omega \tau \\
P_{2}(\omega, t)=\frac{1}{4 \pi^{2}} \frac{e^{2} \mu \omega}{c^{2}} v\left[1-\frac{1}{n^{2} \beta^{2}}\right] \int_{-\infty}^{\infty} d \tau \frac{\sin n \omega \beta\left|\frac{a}{v}+\tau\right|}{\left|\frac{a}{v}+\tau\right|} \cos \omega \tau \\
P_{3}(\omega, t)=\frac{1}{4 \pi^{2}} \frac{e^{2} \mu \omega}{c^{2}} v\left[1-\frac{1}{n^{2} \beta^{2}}\right] \int_{-\infty}^{\infty} d \tau \frac{\sin n \omega \beta\left|\frac{a}{v}-\tau\right|}{\left|\frac{a}{v}-\tau\right|} \cos \omega \tau .
\end{gathered}
$$

The formula (14) contains the known integral:

$$
J_{1}=\int_{-\infty}^{\infty} d \tau \frac{\sin n \omega \beta \tau}{\tau} \cos \omega \tau=\left\{\begin{array}{ll}
\pi ; & n \beta>1 \\
0 ; & n \beta<1
\end{array} .\right.
$$

Formulae (15) and (16) contain the following integrals:

$$
J_{2}=\int_{-\infty}^{\infty} d \tau \frac{\sin n \omega \beta\left|\frac{a}{v}+\tau\right|}{\left|\frac{a}{v}+\tau\right|} \cos \omega \tau
$$

and

$$
J_{3}=\int_{-\infty}^{\infty} d \tau \frac{\sin n \omega \beta\left|\frac{a}{v}-\tau\right|}{\left|\frac{a}{v}-\tau\right|} \cos \omega \tau .
$$

Using the integral (17) we finally get the power spectral formula $P_{1}$ of the produced photons:

$$
P_{1}(\omega, t)=\frac{e^{2}}{4 \pi} \frac{\mu \omega}{c^{2}} v\left[1-\frac{1}{n^{2} \beta^{2}}\right] ; \quad n \beta>1
$$

and

$$
P_{1}(\omega, t)=0 ; \quad n \beta<1 .
$$

Using transformations 


$$
\frac{a}{v}+\tau=T, \quad \frac{a}{v}-\tau=T,
$$

we get after evaluations of the corresponding integrals $J_{2}, J_{3}$ the corresponding spectral formulas $P_{2}, P_{3}$ :

$$
P_{2}(\omega, t)=\frac{e^{2}}{4 \pi} \frac{\mu \omega}{c^{2}} \cos \left(\frac{\omega a}{v}\right) v\left[1-\frac{1}{n^{2} \beta^{2}}\right]=P_{3} ; \quad n \beta>1
$$

and

$$
P_{2}(\omega, t)=P_{3}(\omega, t)=0 ; \quad n \beta<1 .
$$

The sum of the partial spectral formula form the total radiation emitted by the Cerenkov mechanism of the two-charge system:

$$
P(\omega, t)=2\left(P_{1}+P_{2}\right)=\cos ^{2}\left(\frac{a \omega}{2 v}\right) \frac{e^{2}}{4 \pi} \frac{\mu \omega}{c^{2}} v\left[1-\frac{1}{n^{2} \beta^{2}}\right] ; n \beta>1
$$

and

$$
P(\omega, t)=0 ; \quad n \beta<1 .
$$

The zero point of function $P(\omega, t)$ are as follows:

$$
\omega_{0}=0 ; \quad \frac{\omega_{n} a}{2 v}=\frac{(2 n-1)}{2} \pi ; \quad n=1,2,3, \ldots
$$

From the last equation follows

$$
a=\frac{(m-n) 2 \pi v}{\left(\omega_{m}-\omega_{n}\right)}=l \sqrt{1-\frac{v^{2}}{c^{2}}}
$$

or,

$$
l=\frac{2 \pi v}{\sqrt{1-\frac{v^{2}}{c^{2}}}} \frac{(m-n)}{\left(\omega_{m}-\omega_{n}\right)} .
$$

If we know the $n$-th and $m$-th zero points with the corresponding $\omega$-s and velocity of the charges we can exactly determine their rest distance. Then, the rest distance determined by the formula (29) can be compared with the rest distance of the charges obtained by direct measurement and in such a way we can verify the Lorentz contraction.

\section{A feasible experiment}

With regard to the situation in laboratories where the great accelerator works for instance in Grenoble, DESY, CERN and SLAC we can suggest a feasible experiment for the verification of the Lorentz contraction. The experiment must be based on the definition of the length. Instead of two electrons we can consider two bunches with $10^{10}$ electrons in volume $300 \mu \mathrm{m} \times 40 \mu \mathrm{m} \times 0,01 \mathrm{~m}$ with the rest distance $l=1 \mathrm{~m}$. After acceleration 
of the considered bunches the distance of the two bunches is the relativistic length $a$ and it can be determined by the Čerenkov spectrum derived in our article. However, during the acceleration the motion of particles in storage rings is influenced by various kinds of perturbations. It is necessary to consider phenomena such as the ground motion, power supply ripple, noise caused by the quantum emission of synchrotron radiation and noise in the radiofrequency (rf) system and so on. Therefore it is necessary to include the stochasticity caused by these effects in the calculation of the beam dynamics. The stochastic forces can change the distance of the bunches. So instead of the determination of the rest length at the beginning of the experiment, it is more suitable to determine the rest length immediately after the determination of the Cerenkov spectrum.

We can slow down the velocity of bunches by the simultaneous deceleration of every bunch in order to get the final nonrelativistic velocity $v_{f}$ instead of the relativistic velocity $v$ in the spectral formula. It can be performed by switching the electric field or by the sufficiently intensive laser field of photons moving in the opposite direction of motion of the bunches. The simultaneity is the inevitable condition in order to conserve the length during deceleration.

If a particle is accelerated in the system $S$ by the constant acceleration $w$, then the law of its motion with the initial conditions $x(0)=0, v(0)=0$ is as follows:

$$
x_{1}(t)=\frac{c^{2}}{w}\left[\sqrt{1+\left(\frac{w t}{c}\right)^{2}}-1\right]
$$

and in case of the initial condition $x(0)=l, v(0)=0$, we have for the law of its motion

$$
x_{2}(t)=x_{1}(t)+l
$$

So, in case of acceleration of the free two-body system we get:

$$
x_{2}(t)-x_{1}(t)=l
$$

and the observer in the system $S$ observes the distance of the the electrons is equal to $l$. In case the acceleration is replaced by the deceleration, the final result is the same. Or, the observer in the system $S$ finds that the distance of the two electrons or bunches does not change during the deceleration. In case of application of the laser field the simultaneity is broken with the difference $l / c \approx 10^{-9} \mathrm{~s}$ in the system of bunches, for the distance $l=$ $1 \mathrm{~m}$. However, such deviation from the simultaneity is sufficiently small in order not to influence substantially the result of experiment. It is evident that in order the experiment to be meaningful it will be necessary to respect the law of of deceleration motion from which eq. (32) follows.

Our situation does not represents the rigid motion considered by Rindler (1977). He shows that for so called rigid motion at every instant $t=$ constant the two points are separated by a coordinate distance $d x$ inversely proportional to their $\gamma$-factor, and consequently the element bounded by these points 'moves rigidly'.

The two bunches impinge into detector with the time difference $\Delta t=l / v_{f}$. This time difference can be determined by the scintillation detector with the sufficient time resolution. The scintillation detectors or counters consist of scintillationg materials, usually a doped plastic, that emit light in response to molecular excitation by the passage of a 
charged particle. The scintillation light can be detected with photomultipliers or photodiodes. The light yield in a plastic scintillator is usually sufficiently large. The scintillation counters range is size from very small to very large a few square meters. The important feature of scintillation counters is their speed which is in nanosecond range. So they can accurately measure the time of arrival of a charged particle and therefore the speed of a particle (Kleinknecht, 1977).

The rest length measured by the scintillation detector $l=\Delta t v_{f}$ can be compared with the formula (29) in order to verify the Lorentz contraction. For velocity $v_{f}=10^{4} \mathrm{~m}$, we have the $\Delta t \approx 10^{-4} \mathrm{~s}$ with the assumption that the Lorentz contraction corresponding to this velocity can be neglected. To our knowledge the detectors have better time resolution than the calculated $\Delta t$. So, the verification of the Lorentz formula is in principle possible.

\section{Discussion}

We have demonstrated that in case of the system of two equal charges, the Lorentz contraction can be determined from the spectral formula of the Cerenkov radiation. Obviously this effect can be involved into the group of the classical relativistic effects. In case of the system of opposite charges, or, in other words, of the dipole we have instead of $\cos (\omega a / 2 v)$ function $\sin (\omega a / 2 v)$ in the final formulae. To our knowledge the determination of the Lorentz contraction using the Cerenkov effect was not considered in theory and in experiment. After performing the experiment with the Cerenkov radiation of the system of the two charges it will be definitely confirmed the Lorentz contraction.

While the simultaneous acceleration of the system of the two equal charges can be performed immediately in every laboratory with the circle accelerator, the simultaneous acceleration of the system of two opposite charges can be performed only with the laser accelerator. In this equipment the opposite charges are accelerated at the same acceleration as a result of the Compton effect.

The experiment suggested by us is feasible in the sense that the bunches of charges are prepared in every circle accelerator and therefore it is not necessary to prepare substantially new arrangement of equipments for verification of the Lorentz contraction. We hope that sooner or later such experiment will be performed.

\section{REFERENCES}

Ben-Ya'acov, Uri. (2019). Gödel's incompleteness theorem and universal physical theories, arXiv:1906.02724v2 [physics.hist-ph] 12 Jul 2019.

Bolotovskii, B. M. (2009). Vavilov-Čerenkov radiation: its discovery and application, Physics - Uspekhi 52(11), 1099 - 1110.

Cavalleri, G. and Tonni, E., (2000). Comment on Čerenkov effect and the Lorentz contraction, Phys. Rev. A 61, 026101.

Čerenkov, P. A. (1934). The visible radiation of pure liquids caused by $\gamma$-rays, Comptes Rendus Hebdomaclaires des Seances de l' Academic des Sciences USSR 2, 451.

Dittrich, W. (1978). Source Methods in Quantum Field Theory, Fortschritte der Physik 
26,289 .

Dreissler, R. J., (2005). The appearance, apparent speed, and removal of optical effects for relativistically moving objects, Am. J. Phys. 73 (7), July.

Einstein, A., (1916). Relativity: The Special and General Theory,

Publisher: Methuen \& Co Ltd, First Published: December, 1916.

Translated: Robert W. Lawson (Authorised translation), Transcription/Markup: Brian Basgen Convertion to PDF: Sjoerd Langkemper,

Offline Version: Einstein Reference Archive (marxists.org) 1999.

Einstein, A., (1919). Über die spezielle und die allgemeine relativitäts Theorie, Vierte Auflage, Vieweg \& Sohn, Braunschweig.; chapter 9.

Fok, V., (1961). Theory of space, time and gravity, GIFML, Moscow.

I. M. Frank, I. M. (1942). Izv. Acad. Nauk USSR, Ser. Fiz. 6, 3.

Frank I.M. (1946). Radiation of Electrons Moving in Medium with Superlight Velocity Usp. Fiz. Nauk, 30, No 3-4, pp. 149-183.

Frank, I. M. (1952). in: Vavilov Memorial Volume (Izd. Acad. Nauk USSR, 1952) 172.

Frank, I. M. (1984). The Čerenkov-Vavilov radiation for the electric and magnetic multipoles, Uspekhi Fiz. Nauk No. 144, 251-275.

Ginzburg, V. L. (1940). ZhETF 10, 589.

Ginzburg, V. L. (1986).Radiation by uniformly moving sources, in: The lessons of Quantum Theory, ed. by J. DeBoer, E. Dal and O. Ulfbeck (Elsevier Science Publishers, B.V., 1986) and references therein.

Heaviside, O. (1889). On the electromagnetic effects due to the motion of electrification through a dielectric, Philos. Mag., S. 5, 27, 324-339.

Kelvin, L. (1901). Nineteenth century clouds over the dynamical theory of heat and light, Philos. Mag., S. 6, 2, 1-40.

Kleinknecht, K. (1986). Detectors for Particle Radiation, (Cambridge Univ. Press, 1986).

Lampa, A., (1924). Wie erscheint nach der Relativitätstheorie ein bewegter Stab einem ruhenden Beobachter? (How does a moving rod appear for an observer at rest according to the theory of relativity?), Z. Phys. 27, 138-148.

Mallet, L. (1926). Spectral research of luminescence of water and other media with gamma radiation, Comptes Rendus, 183, 274.; ibid. (1929a). Comptes Rendus, 187, 222.; ibid. (1929b). Comptes Rendus, 188, 445.

Pardy, M. (1969). A remark on the clock paradox, Physics Letters A 28, (11), 10 March 1969, 766-767.

Pardy, M. (1983). Particle production by the Cerenkov mechanism, Phys. Lett. A 94, No. $1,30$.

Pardy, M. (1989). Finite-temperature Čerenkov radiation, Physics Letters A 134 (6), 357. 
Pardy, M. (1997). Čerenkov effect and the Lorentz contraction, Phys. Rev. A 55, 1647.

Pardy, M. (2002). Čerenkov effect with massive photons, International Journal of Theoretical Physics, 41 (5), 887.

Pardy, M. (2000). The Čerenkov-Compton effect in particle physics, arXiv:hep-ph/0009063v2.

Pardy, M. (2004). Compton effect in dielectric medium, arXiv:hep-ph/0406008v1.

Pardy, M. (2007). Missing experiment in relativity and gravity, arXiv: 0710.3489v2 [nuclex] 8 Feb 2008 .

Penrose, R., (1959). The apparent shape of a relativistically moving sphere, Proc. Cambridge Philos. Soc. 55, 137-139.

Rindler, W. (1977). Essential Relativity, (Springer-Verlag, New York, 1977).

Schwinger, J., Tsai, W. Y. and Erber, T. (1976). Classical and quantum theory of synergic synchrotron-Čerenkov radiation, Annals of Physics (NY) 96, 303.

Sommerfeld, A. (1904). Zur Elektronentheorie: II. Grundlagen für eine allgemeine Dynamik des Elektrons, Göttingen Nachr., 99, 363-439.

Terrell, J. (1959). The invisibility of the Lorentz contraction, Phys. Rev. 116, 1041 (1959).

Tamm, I. E. and Frank, I. M. (1937). The coherent radiation of a fast electron in a medium, Dokl. Akad. Nauk SSSR 14, 109.

Weisskopf, V. F. (1960). The visual appearance of rapidly moving objects, Physics Today, September (1960). 13, 24. 